
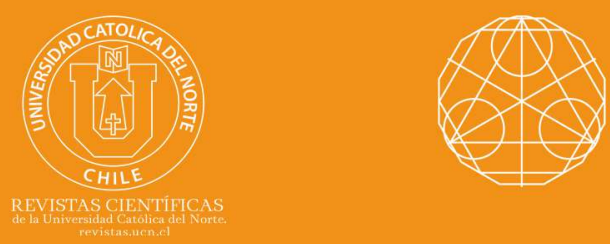

\title{
Regularity and normality in ideal bitopological spaces
}

Néstor Raúl Pachón Rubianoํㅜ orcid.org/0000-0002-7191-127X

${ }^{1}$ Escuela Colombiana de Ingeniería Julio Garavito, Depto. de Matemáticas, Bogotá, Colombia.

nestor.pachon@escuelaing.edu.co

\section{Abstract:}

We introduce, and study, the regularity and normality in ideal bitopological spaces, absent subject in literature. Our definitions have the advantage of using only the open sets of the two underlying topologies. These new concepts represent generalizations of Kelly's concepts of pairwise regularity and pairwise normality. The extension of the $T_{0}, T_{1}$ and $T_{2}$ axioms to these spaces is due to Caldas et al.

Keywords: Bitopological spaces; Ideal bitopological spaces; Separation in bitopological spaces; Separation in ideal bitopological spaces.

MSC (2020): 54D10.

Cite this article as (IEEE citation style):

N. R. Pachón Rubiano, "Regularity and normality in ideal bitopological spaces ", Proyecciones (Antofagasta, On line), vol. 40, no. 2, pp. 425-445, 2021, doi: 10.22199/issn.0717-62792021-02-0026

Article copyright: (C) 2021 Néstor Raúl Pachón Rubiano. This is an open access article distributed under the terms of the Creative Commons License, which permits unrestricted use and distribution provided the original author and source are credited. 


\section{Introduction and preliminaries}

Bitopological spaces were introduced in 1963 by J. Kelly [2], as a tool to systematize the study of quasi-metrics. The study of the axioms of separation in this type of spaces was started by him, and has had important contributions from I. Reilly [7]. Regarding the study of the axioms of separation in ideal bitopological spaces, Caldas, Jafari, Popa and Rajesh, in a joint work of 2010, have defined what refers to the axioms $T_{0}, T_{1}$ and $\mathrm{T}_{2}$. With respect to extensions of the axioms of regularity and normality in these spaces, we have no references.

In this paper we introduce and investigate the pairwise $\mathcal{I}$-regular spaces, the pairwise $\mathcal{I}$-normal spaces, the strongly pairwise $\mathcal{I}$-regular spaces and the strongly pairwise $\mathcal{I}$-normal spaces, and for this we only use the open sets of the underlying topologies. This is not only more natural but it allows us to work in a simpler way.

An ideal $\mathcal{I}$ in a set $X$ is a subset of $\mathcal{P}(X)$, the power set of $X$, such that: $(i)$ if $A \subseteq B \subseteq X$ and $B \in \mathcal{I}$ then $A \in \mathcal{I}$, and (ii) if $\{A, B\} \subseteq \mathcal{I}$ then $A \cup B \in \mathcal{I}$.

Some useful ideals in $X$ are: $(i) \mathcal{P}(A)$, where $A \subseteq X,($ ii $) \mathcal{I}_{f}(X)$, the ideal of all finite subsets of $X,($ iii $) \mathcal{I}_{c}(X)$, the ideal of all countable subsets of $X$ and $(i v) \mathcal{I}_{n}(X)$, the ideal of all nowhere dense sets in a topological space $(X, \tau)$. If $\mathcal{I}$ is an ideal in $X$ and if $f: X \rightarrow Y$ is a function, then the set $f(\mathcal{I})=\{f(I): I \in \mathcal{I}\}$ is an ideal in $Y$ [3]. Furthermore, if $\mathcal{J}$ is an ideal in $Y$ and if $f: X \rightarrow Y$ is an one-one function, then the set $f^{-1}(\mathcal{J})=\left\{f^{-1}(J): J \in \mathcal{J}\right\}$ is an ideal in $X$ [3]. If $\mathcal{J}$ is an ideal in $Y$ and if $f: X \rightarrow Y$ is a function, then the set $\mathcal{I}_{f, \mathcal{J}}=\left\{A \subseteq X\right.$ : there is a $J \in \mathcal{J}$ with $\left.A \subseteq f^{-1}(J)\right\}$ is an ideal in $X[6]$. If $\mathcal{I}$ is an ideal in $X$ and $A \subseteq X$, then the set $\mathcal{I}_{A}=\{A \cap I: I \in \mathcal{I}\}$ is an ideal in $A$.

If $(X, \tau)$ is a topological space and $\mathcal{I}$ is an ideal in $X$, then $(X, \tau, \mathcal{I})$ is called an ideal space. If $(X, \tau)$ is a topological space and $A \subseteq X$ then the closure and the interior of $A$ are denoted by $\bar{A}$ (or $a d h_{\tau}(A)$, or $a d h(A)$ ) and $\AA\left(\right.$ or $\operatorname{int}_{\tau}(A)$, or $\left.\operatorname{int}(A)\right)$, respectively.

An ideal space $(X, \tau, \mathcal{I})$ is said to be $\mathcal{I}$-compact [3] if for each open cover $\left\{V_{\alpha}\right\}_{\alpha \in \Delta}$ of $X$, there is a finite $\Delta_{0} \subseteq \Delta$ such that $X \backslash \bigcup_{\alpha \in \Delta_{0}} V_{\alpha} \in \mathcal{I}$.

Given an ideal space $(X, \tau, \mathcal{I})$ and a set $A \subseteq X$, we denote by $A^{*}(\mathcal{I})=$ $\{x \in X: U \cap A \notin \mathcal{I}$, for every $U \in \tau$ with $x \in U\}$, written simply as $A^{*}$ when there is no chance for confusion. It is clear that $A^{*} \subseteq \bar{A}$. A Kuratowski closure operator [8] for a topology $\tau^{*}(\mathcal{I})$, finer than $\tau$, is defined by 
$C l^{*}(A)=A \cup A^{*}$, for all $A \subseteq X$. When there is no chance for confusion $\tau^{*}(\mathcal{I})$ is denoted by $\tau^{*}$. The topology $\tau^{*}$ has as a base $\beta(\tau, \mathcal{I})=$ $\{V \backslash I: V \in \tau$ and $I \in \mathcal{I}\}[1]$.

If $\mathcal{I}$ is an ideal in $X$ and $\mathcal{J}$ is an ideal in $Y$, then $\mathcal{I} \otimes \mathcal{J}$ [5] is the set of all $D \subseteq X \times Y$ such that there exist $I \in \mathcal{I}, A \subseteq X, J \in \mathcal{J}$ and $B \subseteq Y$, with $D \subseteq(A \times J) \cup(I \times B)$. It is shown in [5] that $\mathcal{I} \otimes \mathcal{J}$ is an ideal in $X \times Y$.

If $\left\{X_{i}: i \in \Lambda\right\}$ is a collection of sets and if $\mathcal{I}_{i}$ is an ideal in $X_{i}$, for each $i \in \Lambda$, we will denote by $\bigotimes_{i \in \Lambda} \mathcal{I}_{i}$ the set of all $A \subseteq \prod_{i \in \Lambda} X_{i}$ such that there exists a finite $\Lambda_{0} \subseteq \Lambda$ with $A \subseteq \bigcup_{i \in \Lambda_{0}} p_{i}^{-1}\left(I_{i}\right)$, for some $I_{i} \in \mathcal{I}_{i}$, for each $i \in \Lambda_{0}[6]$. Here $p_{i}$ represents the $i$-th projection. It is very simple to prove that $\bigotimes_{i \in \Lambda} \mathcal{I}_{i}$ is an ideal in $\prod_{i \in \Lambda} X_{i}$.

If $\tau$ and $\beta$ are topologies in a set $X$, then $(X, \tau, \beta)$ is called a bitopological space [2]. If, in addition, $\mathcal{I}$ is an ideal in $X$, then $(X, \tau, \beta, \mathcal{I})$ is an ideal bitopological space.

Finally, throughout this work we will use the following topologies in $\mathbf{R}$ : a) $\mathcal{C}=\{\varnothing, \mathbf{R}\} \cup\{(a, \infty): a \in \mathbf{R}\}$, b) $\mathcal{L}$ is the (Sorgenfrey) topology of all $V \subseteq \mathbf{R}$ such that, for each $a \in V$, there is a $b>a$ such that $[a, b) \subseteq V$, c) $\gamma$ is the topology in which the neighborhoods of any nonzero point being as in the usual topology $\mathcal{U}$, while neighborhoods of 0 will have the form $U \backslash F$, where $U$ is a neighborhood of 0 in $\mathcal{U}$ and $F=\left\{1 / n: n \in \mathbf{Z}^{+}\right\}$.

The symbol $\square$ is used to indicate the end of a proof.

\section{Pairwise $\mathcal{I}$-regular spaces}

In this section we introduce our first extension of regularity to ideal bitopological spaces. We present some characterizations and properties of interest. We recall that a bitopological space $\left(X, \tau_{1}, \tau_{2}\right)$ is defined to be pairwise regular [2] if for every $i \in\{1,2\}$, every $\tau_{i}$-closed set $F$ and $x \in X \backslash F$, there are $U \in \tau_{i}$ and $V \in \tau_{j}$, where $j \in\{1,2\} \backslash\{i\}$, such that $x \in U, F \subseteq V$ and $U \cap V=\varnothing$. The concept that we will present here is a generalization of that definition.

Definition 2.1 The ideal bitopological space $\left(X, \tau_{1}, \tau_{2}, \mathcal{J}\right)$ is pairwise $\mathcal{I}$ -regular if, for every $i \in\{1,2\}$, every $\tau_{i}$-closed set $F$ and $x \in X \backslash F$, there exist $U \in \tau_{i}$ and $V \in \tau_{j}$, where $j \in\{1,2\} \backslash\{i\}$, such that $x \in U, F \backslash V \in \mathcal{J}$ and $U \cap V=\varnothing$. 
It is clear that if $\left(X, \tau_{1}, \tau_{2}\right)$ is pairwise regular then $\left(X, \tau_{1}, \tau_{2}, \mathcal{J}\right)$ is pairwise $\mathcal{I}$-regular, and that $\left(X, \tau_{1}, \tau_{2}\right)$ is pairwise regular if and only if $\left(X, \tau_{1}, \tau_{2},\{\varnothing\}\right)$ is pairwise $\mathcal{I}$-regular.

Example 2.2 1) The space $(\mathbf{R}, \mathcal{C}, \mathcal{L}, \mathcal{J})$, where $\mathcal{J}$ is the ideal of all upper bounded subsets of $\mathbf{R}$, is pairwise $\mathcal{I}$-regular, because:

a) If $F$ is $\mathcal{C}$-closed and if $a \in \mathbf{R} \backslash F$ then there exists $\alpha<a$ such that $(\alpha, \infty) \subseteq \mathbf{R} \backslash F$. If we do $U=\left(\frac{\alpha+a}{2}, \infty\right)$ and $V=\left(-\infty, \frac{\alpha+a}{2}\right)$ then $U \in \mathcal{C}$, $V \in \mathcal{L}, a \in U, F \backslash V=\varnothing \in \mathcal{J}$ and $U \cap V=\varnothing$.

b) If $G$ is $\mathcal{L}$-closed and if $a \in \mathbf{R} \backslash G$ then there is a $b>a$ such that $[a, b) \subseteq \mathbf{R} \backslash G$. If we do $V=(b, \infty)$ and $U=[a, b)$, then $V \in \mathcal{C}, U \in \mathcal{L}$, $U \cap V=\varnothing$ and $G \backslash V \in \mathcal{J}$.

However $(\mathbf{R}, \mathcal{C}, \mathcal{L})$ is not pairwise regular. In fact, the set $G=(-\infty, 0]$ is $\mathcal{L}$-closed, and if $U \in \mathcal{C}, V \in \mathcal{L}, G \subseteq U$ and $1 \in V$, it is clear that $U=\mathbf{R}$ and so $U \cap V \neq \varnothing$.

2) If $\tau=\{\varnothing, \mathbf{R}\}, \beta=\mathcal{P}(\mathbf{R})$ and if $\mathcal{J}=\mathcal{I}_{c}(\mathbf{R})$, then $(\mathbf{R}, \tau, \beta, \mathcal{J})$ is not pairwise $\mathcal{I}$-regular. In fact, the set $F=\mathbf{R} \backslash\{1\}$ is $\beta$-closed, and if $U \in \tau$, $V \in \beta, F \backslash U \in \mathcal{J}$ and if $1 \in V$, then the only option is $U=\mathbf{R}$, and so $U \cap V=V \neq \varnothing$.

We will begin by presenting some characterizations of pairwise $\mathcal{I}$-regularity.

Theorem 2.3 The following propositions are equivalent:

1) The space $\left(X, \tau_{1}, \tau_{2}, \mathcal{J}\right)$ is pairwise $\mathcal{I}$-regular.

2) For all $i \in\{1,2\}$, every $W \in \tau_{i}$ and $x \in W$, there is a $U \in \tau_{i}$ such that $x \in U$ and $a d h_{\tau_{j}}(U) \backslash W \in \mathcal{I}$, where $j \in\{1,2\} \backslash\{i\}$.

3) For every $i \in\{1,2\}$, every $\tau_{i}$-closed set $F$ and $x \in X \backslash F$, there exist $U \in \tau_{i}$ such that $x \in U$ and $a d h_{\tau_{j}}(U) \cap F \in \mathcal{I}$.

Proof. 1) $\rightarrow$ 2) Suppose that $i \in\{1,2\}, W \in \tau_{i}$ and $\quad x \in W$. We can find a $U \in \tau_{i}$ and a $V \in \tau_{j}$, where $j \neq i$, such that $x \in U,(X \backslash W) \backslash V \in \mathcal{J}$ and $U \cap V=\varnothing$. Hence $a d h_{\tau_{j}}(U) \subseteq X \backslash V$ and so $a d h_{\tau_{j}}(U) \backslash W \in \mathcal{J}$.

$2) \rightarrow 3$ ) Suppose that $i \in\{1,2\}, F$ is a $\tau_{i}$-closed set and $x \in X \backslash F$. There is a $U \in \tau_{i}$ such that $x \in U$ and $a d h_{\tau_{j}}(U) \backslash(X \backslash F) \in \mathcal{J}$, where $j \neq i$. Thus $a d h_{\tau_{j}}(U) \cap F \in \mathcal{J}$.

$3) \rightarrow 1$ ) If $i \in\{1,2\}, F$ is a $\tau_{i}$-closed set and $x \in X \backslash F$, there exists $U \in \tau_{i}$ such that $x \in U$ and $a d h_{\tau_{j}}(U) \cap F \in \mathcal{J}$, where $j \neq i$. Then $F \backslash\left(X \backslash a d h_{\tau_{j}}(U)\right) \in \mathcal{J}$, with $U \cap\left[X \backslash a d h_{\tau_{j}}(U)\right]=\varnothing$.

Now we will show a relationship between pairwise $\mathcal{I}$-regularity and compactness. 
Theorem 2.4 If the space $\left(X, \tau_{1}, \tau_{2}, \mathcal{J}\right)$ is pairwise $\mathcal{I}$-regular and if $i \neq j$, then for every $\tau_{i}$-compact set $K$ and every $\tau_{i}$-closed set $F$, with $F \cap K=\varnothing$, there are disjoint $U \in \tau_{i}$ and $V \in \tau_{j}$, such that $K \subseteq U$ and $F \backslash V \in \mathcal{J}$.

Proof. Suppose that $i \neq j, K$ is a $\tau_{i}$-compact set, $F$ is a $\tau_{i}$-closed set and $F \cap K=\varnothing$. For each $x \in K$, there are disjoint $U_{x} \in \tau_{i}$ and $V_{x} \in \tau_{j}$ such that $x \in U_{x}$ and $F \backslash V_{x} \in \mathcal{J}$. There is a finite $K_{0} \subseteq K$ such that $K \subseteq U=$ $\bigcup_{x \in K_{0}} U_{x} \in \tau_{i}$. If $V=\bigcap_{x \in K_{0}} V_{x}$ then $V \in \tau_{j}, F \backslash V=\bigcup_{x \in K_{0}}\left(F \backslash V_{x}\right) \in \mathcal{J}$ and $U \cap V=\varnothing$.

The next two properties have to do with the products of pairwise $\mathcal{I}$ regular spaces.

Theorem 2.5 If $\left(X, \tau_{1}, \tau_{2}, \mathcal{J}\right)$ and $\left(Y, \beta_{1}, \beta_{2}, \mathcal{L}\right)$ are pairwise $\mathcal{I}$-regular spaces, then $\left(X \times Y, \tau_{1} \times \beta_{1}, \tau_{2} \times \beta_{2}, \mathcal{J} \otimes \mathcal{L}\right)$ is pairwise $\mathcal{I}$-regular.

Proof. Suppose that $i \in\{1,2\}, W \in \tau_{i} \times \beta_{i}$ and $(a, b) \in W$. There are $U \in \tau_{i}$ and $V \in \beta_{i}$ such that $(a, b) \in U \times V \subseteq W$. Now, by Theorem 2.3, there are $U_{1} \in \tau_{i}$ and $V_{1} \in \beta_{i}$ such that $a \in \bar{U}_{1}, b \in V_{1}, a d h_{\tau_{j}}\left(U_{1}\right) \backslash U \in \mathcal{J}$ and $a d h_{\beta_{j}}\left(V_{1}\right) \backslash V \in \mathcal{L}$, where $j \neq i$. Hence $a d h_{\tau_{j} \times \beta_{j}}\left(U_{1} \times V_{1}\right) \backslash W \subseteq$ $\left[a d h_{\tau_{j}}\left(U_{1}\right) \times a d h_{\beta_{j}}\left(V_{1}\right)\right] \backslash(U \times V)=\left[\left(a d h_{\tau_{j}}\left(U_{1}\right) \backslash U\right) \times a d h_{\beta_{j}}\left(V_{1}\right)\right] \cup$ $\left[a d h_{\tau_{j}}\left(U_{1}\right) \times\left(a d h_{\beta_{j}}\left(V_{1}\right) \backslash V\right)\right]$, and so $a d h_{\tau_{j} \times \beta_{j}}\left(U_{1} \times V_{1}\right) \backslash W \in \mathcal{J} \otimes \mathcal{L}$. Thus $\left(X \times Y, \tau_{1} \times \beta_{1}, \tau_{2} \times \beta_{2}, \mathcal{J} \otimes \mathcal{L}\right)$ is pairwise $\mathcal{I}$-regular, by Theorem 2.3 .

Theorem 2.6 If $\left\{\left(X_{\alpha}, \tau_{1 \alpha}, \tau_{2 \alpha}, \mathcal{J}_{\alpha}\right): \alpha \in \Delta\right\}$ is a collection of pairwise $\mathcal{I}$-regular spaces, then $\left(\prod_{\alpha \in \Delta} X_{\alpha}, \prod_{\alpha \in \Delta} \tau_{1 \alpha}, \prod_{\alpha \in \Delta} \tau_{2 \alpha}, \bigotimes_{\alpha \in \Delta} \mathcal{J}_{\alpha}\right)$ is pairwise $\mathcal{I}$ -regular.

Proof. To begin, we define $X=\prod_{\alpha \in \Delta} X_{\alpha}, \tau_{1}=\prod_{\alpha \in \Delta} \tau_{1 \alpha}$ and $\tau_{2}=\prod_{\alpha \in \Delta} \tau_{2 \alpha}$.

Suppose that $i \in\{1,2\}, W \in \tau_{i}$ and $a=\left(a_{\alpha}\right)_{\alpha \in \Delta} \in W$. There are $\left\{\alpha_{1}, \alpha_{2}, \ldots, \alpha_{r}\right\} \subseteq \Delta$ and $V_{\alpha_{j}} \in \tau_{i \alpha_{j}}$, for each $j \in\{1,2, \ldots, r\}$, such that $a \in$ $\bigcap_{j=1}^{r} p_{\alpha_{j}}^{-1}\left(V_{\alpha_{j}}\right) \subseteq W$, where $p_{\alpha_{j}}$ is the $\alpha_{j}-t h$ projection. If $j \in\{1,2, \ldots, r\}$ then $a_{\alpha_{j}} \in V_{\alpha_{j}}$, and so, by Theorem 2.3, there is a $U_{\alpha_{j}} \in \tau_{i \alpha_{j}}$ such that $a_{\alpha_{j}} \in U_{\alpha_{j}}$ and $a d h_{\tau_{k \alpha_{j}}}\left(U_{\alpha_{j}}\right) \backslash V_{\alpha_{j}} \in \mathcal{J}_{\alpha_{j}}$, where $k \in\{1,2\} \backslash\{i\}$. Now, 
since each function $p_{\alpha_{j}}:\left(X, \tau_{i}\right) \rightarrow\left(X_{\alpha_{j}}, \tau_{i \alpha_{j}}\right)$ is continuous, we have that $U=\bigcap_{j=1}^{r} p_{\alpha_{j}}^{-1}\left(U_{\alpha_{j}}\right) \in \tau_{i}$. Moreover $a \in \bigcap_{j=1}^{r} p_{\alpha_{j}}^{-1}\left(U_{\alpha_{j}}\right)$.

On the other hand, $a d h_{\tau_{k}}(U) \backslash W \subseteq \bigcap_{j=1}^{r} a d h_{\tau_{k}}\left(p_{\alpha_{j}}^{-1}\left(U_{\alpha_{j}}\right)\right) \backslash \bigcap_{j=1}^{r} p_{\alpha_{j}}^{-1}\left(V_{\alpha_{j}}\right)$ $\subseteq \bigcap_{j=1}^{r} p_{\alpha_{j}}^{-1}\left(a d h_{\tau_{k \alpha_{j}}}\left(U_{\alpha_{j}}\right)\right) \backslash \bigcap_{j=1}^{r} p_{\alpha_{j}}^{-1}\left(V_{\alpha_{j}}\right) \subseteq \bigcup_{j=1}^{r}\left[p_{\alpha_{j}}^{-1}\left(a d h_{\tau_{k \alpha_{j}}}\left(U_{\alpha_{j}}\right) \backslash V_{\alpha_{j}}\right)\right]$ and so $a d h_{\tau_{k}}(U) \backslash W \in \bigotimes_{\alpha \in \Delta} \mathcal{J}_{\alpha}$, because $p_{\alpha_{j}}^{-1}\left(a d h_{\tau_{k \alpha_{j}}}\left(U_{\alpha_{j}}\right) \backslash V_{\alpha_{j}}\right) \in \bigotimes_{\alpha \in \Delta} \mathcal{J}_{\alpha}$, for each $j \in\{1,2 . ., r\}$.

By Theorem 2.3, $\left(X, \tau_{1}, \tau_{2}, \underset{\alpha \in \Delta}{\bigotimes} \mathcal{J}_{\alpha}\right)$ is pairwise $\mathcal{I}$-regular.

In the three theorems that follow we will present some functional properties of pairwise $\mathcal{I}$-regular spaces.

Theorem 2.7 If for each $i \in\{1,2\}$ we have that $f:\left(X, \tau_{i}\right) \rightarrow\left(Y, \beta_{i}\right)$ is a continuous, open, closed and surjective function, and if $\left(X, \tau_{1}, \tau_{2}, \mathcal{J}\right)$ is pairwise $\mathcal{I}$-regular, then the space $\left(Y, \beta_{1}, \beta_{2}, f(\mathcal{J})\right)$ pairwise $\mathcal{I}$-regular.

Proof. Suppose that $i \in\{1,2\}, W \in \beta_{i}$ and that $f(a) \in W$. Since $a \in f^{-1}(W)$ and $f^{-1}(W) \in \tau_{i}$, there exists $U \in \tau_{i}$ such that $a \in U$ and $a d h_{\tau_{j}}(U) \backslash f^{-1}(W) \in \mathcal{J}$, where $j \neq i$. Hence $f\left[a d h_{\tau_{j}}(U) \backslash f^{-1}(W)\right] \in$ $f(\mathcal{J})$. If we now consider that $f$ is continuous, closed and surjective, thus $a d h_{\beta_{j}}(f(U)) \backslash W=f\left(a d h_{\tau_{j}}(U)\right) \backslash W=f\left(a d h_{\tau_{j}}(U)\right) \backslash f\left(f^{-1}(W)\right) \subseteq$ $f\left[a d h_{\tau_{j}}(U) \backslash f^{-1}(W)\right]$, and so $a d h_{\beta_{j}}(f(U)) \backslash W \in f(\mathcal{J})$, with $f(U) \in \beta_{i}$ and $f(a) \in f(U)$. Theorem 2.3 implies that $\left(Y, \beta_{1}, \beta_{2}, f(\mathcal{J})\right)$ is pairwise $\mathcal{I}$-regular.

Theorem 2.8 If for each $i \in\{1,2\}$ have that $f:\left(X, \tau_{i}\right) \rightarrow\left(Y, \beta_{i}\right)$ is a continuous, closed and injective function, and if $\left(Y, \beta_{1}, \beta_{2}, \mathcal{L}\right)$ is pairwise $\mathcal{I}$-regular, then the space $\left(X, \tau_{1}, \tau_{2}, f^{-1}(\mathcal{L})\right)$ is pairwise $\mathcal{I}$-regular.

Proof. Suppose that $i \in\{1,2\}, F$ is a $\tau_{i}$-closed set and that $a \in X \backslash F$. Given that $f(a) \notin f(F)$ and $f(F)$ is $\beta_{i}$-closed, there are disjoint $W \in \beta_{i}$ and $T \in \beta_{j}$, where $j \neq i$, such that $f(a) \in W$ and $f(F) \backslash T \in \mathcal{L}$. This implies that $a \in f^{-1}(W), F \backslash f^{-1}(T) \in f^{-1}(\mathcal{L}), f^{-1}(W) \in \tau_{i}, f^{-1}(T) \in \tau_{j}$ and $f^{-1}(W) \cap f^{-1}(T)=\varnothing$. 
Definition 2.9 If $\left(X, \tau_{1}, \tau_{2}\right)$ and $\left(Y, \beta_{1}, \beta_{2}\right)$ are bitopological spaces, then a function $f:\left(X, \tau_{1}, \tau_{2}\right) \rightarrow\left(Y, \beta_{1}, \beta_{2}\right)$ is said to be pairwise perfect if, for each $i \in\{1,2\}, f:\left(X, \tau_{i}\right) \rightarrow\left(Y, \beta_{i}\right)$ is a continuous, closed and surjective and if, for each $y \in Y, f^{-1}(\{y\})$ is compact in $\left(X, \tau_{i}\right)$.

Theorem 2.10 If $f:\left(X, \tau_{1}, \tau_{2}\right) \rightarrow\left(Y, \beta_{1}, \beta_{2}\right)$ is pairwise perfect, and if $\mathcal{L}$ is an ideal in $Y$ such that $\left(X, \tau_{1}, \tau_{2}, \mathcal{I}_{f, \mathcal{L}}\right)$ is pairwise $\mathcal{I}$-regular, then $\left(Y, \beta_{1}, \beta_{2}, \mathcal{L}\right)$ is pairwise $\mathcal{I}$-regular space.

Proof. Suppose that $i \in\{1,2\}, H$ is a $\beta_{i}$-closed set and that $b=f(a) \in$ $Y \backslash H$. Since $f^{-1}(\{b\})$ is $\tau_{i}$-compact, $f^{-1}(H)$ is $\tau_{i}$-closed and $f^{-1}(\{b\}) \cap$ $f^{-1}(H)=\varnothing$, Theorem 2.4 implies that there are $U \in \tau_{i}$ and $V \in \tau_{j}$, where $j \neq i$, such that $f^{-1}(\{b\}) \subseteq U, f^{-1}(H) \backslash V \in \mathcal{I}_{f, \mathcal{L}}$ and $U \cap V$ $=\varnothing$. There exists $L \in \mathcal{L}$ with $f^{-1}(H) \backslash V \subseteq f^{-1}(L)$. In this way we obtain that: $f^{-1}(H) \subseteq V \cup f^{-1}(L),(X \backslash V) \cap f^{-1}(Y \backslash L) \subseteq f^{-1}(Y \backslash H)$, $f\left[(X \backslash V) \cap f^{-1}(Y \backslash L)\right] \subseteq Y \backslash H, H \subseteq Y \backslash f\left[(X \backslash V) \backslash f^{-1}(L)\right] \subseteq Y \backslash[f(X \backslash V) \backslash L]=$ $[Y \backslash f(X \backslash V)] \cup L$, and this implies that $H \backslash[Y \backslash f(X \backslash V)] \subseteq L$. Thus $H \backslash[Y \backslash f(X \backslash V)]$ $\in \mathcal{L}$. On the other hand $b \in Y \backslash f(X \backslash U), Y \backslash f(X \backslash U) \in \beta_{i}, Y \backslash f(X \backslash V) \in$ $\beta_{j}$. Given that $f$ is surjective, $[Y \backslash f(X \backslash U)] \cap[Y \backslash f(X \backslash V)]=\varnothing$.

We will end this section by showing two properties of pairwise $\mathcal{I}$-regularity, related to $\tau^{*}$ topologies.

If $\tau$ is a topology in $X$, the collection of all closed sets in $(X, \tau)$ is a base for a topology $\tau^{c}$ in $X$. If, moreover, $\mathcal{J}$ is an ideal in $X$ then the set $\tau \cup \mathcal{J}$ is a base for a topology $\tau \oplus \mathcal{J}$ in $X$, finer than $\tau$ [5]. Another base for $\tau \oplus \mathcal{J}$ is the set $\{V \cup J: V \in \tau$ and $J \in \mathcal{J}\}$.

Theorem 2.11 If $(X, \tau, \mathcal{J})$ an ideal space, then $\left(X, \tau^{*},\left(\tau^{c}\right)^{*}, \mathcal{J}\right)$ is pairwise $\mathcal{I}$-regular.

Proof. a) If $F$ is $\tau^{*}$-closed and if $a \in X \backslash F$ then there exist $V \in \tau$ and $J \in \mathcal{J}$, such that $a \in V \backslash J \subseteq X \backslash F$. So $a \in V, V \in \tau^{*}, V \cap(F \backslash J)=\varnothing$, $F \backslash J \in\left(\tau^{c}\right)^{*}$ and $F \backslash(F \backslash J)=F \cap J \in \mathcal{J}$.

b) If $G$ is $\left(\tau^{c}\right)^{*}$-closed and if $b \in X \backslash G$ then there are $T \in \tau^{c}$ and $L \in \mathcal{J}$, such that $b \in T \backslash L \subseteq X \backslash G$. Now, there is a $W \in \tau$ such that $b \in X \backslash W \subseteq T$. Hence $(X \backslash W) \backslash L \subseteq X \backslash G$, and so $G \backslash W \subseteq L$. In this way $G \backslash W \in \mathcal{J}$ and $b \in X \backslash W$, with $W \in \tau^{*}$ and $X \backslash W \in\left(\tau^{c}\right)^{*}$.

Theorem 2.12 If $(X, \tau, \mathcal{J})$ an ideal space, then $\left(X, \tau^{*} \oplus \mathcal{J},\left(\left(\tau^{*}\right)^{c}\right)^{*}, \mathcal{J}\right)$ is pairwise $\mathcal{I}$-regular. 
Proof. a) If $F$ is $\left(\tau^{*} \oplus \mathcal{J}\right)$-closed and if $a \in X \backslash F$, then there exist $T \in \tau^{*}$ and $J \in \mathcal{J}$ such that $a \in T \cup J \subseteq X \backslash F$.

i) Suppose that $a \in J$. We have that $J \in \tau^{*} \oplus \mathcal{J}, F \backslash[(X \backslash T) \backslash J]=\varnothing \in \mathcal{J}$, $(X \backslash T) \backslash J \in\left(\left(\tau^{*}\right)^{c}\right)^{*}$ and $J \cap[(X \backslash T) \backslash J]=\varnothing$.

ii) Suppose that $a \in T$. There are $V \in \tau$ and $I \in \mathcal{J}$ such that $a \in V \backslash I \subseteq T$. In this way $(V \backslash I) \cup J \subseteq T \cup J \subseteq X \backslash F$, and so $F \backslash[(X \backslash V) \backslash J] \subseteq I \backslash J$. This implies that $F \backslash[(X \backslash V) \backslash J] \in \mathcal{J}$. Moreover $(X \backslash V) \backslash J \in\left(\tau^{c}\right)^{*} \subseteq\left(\left(\tau^{*}\right)^{c}\right)^{*}$, $V \backslash I \in \tau^{*} \oplus \mathcal{J}$ and $(V \backslash I) \cap[(X \backslash V) \backslash J]=\varnothing$.

b) If $G$ is $\left(\left(\tau^{*}\right)^{c}\right)^{*}$-closed and if $a \in X \backslash G$, then there are $T \in\left(\tau^{*}\right)^{c}$ and $J \in \mathcal{J}$ such that $a \in T \backslash J \subseteq X \backslash G$. Now, there exists $R \in \tau^{*}$ with $a \in$ $X \backslash R \subseteq T$. Note that $X \backslash R \in\left(\tau^{*}\right)^{c} \subseteq\left(\left(\tau^{*}\right)^{c}\right)^{*}$. On the other hand, since $(X \backslash R) \backslash J \subseteq X \backslash G$ we have that $G \backslash R \subseteq J$, and so $G \backslash R \in \mathcal{J}$. Moreover $R \in \tau^{*} \oplus \mathcal{J}$.

\section{Pairwise $\mathcal{I}$-normal spaces}

In this section we introduce our first extension of normality to ideal bitopological spaces. We recall that a bitopological space $\left(X, \tau_{1}, \tau_{2}\right)$ is defined to be pairwise normal [2] if for any disjoint pair of a $\tau_{i}$-closed set $F$ and a $\tau_{j}$-closed set $G$, with $i \neq j$, there are disjoint $U \in \tau_{j}$ and $V \in \tau_{i}$ such that $F \subseteq U$ and $G \subseteq V$. Our concept is a generalization of that definition.

Definition 3.1 The ideal bitopological space $\left(X, \tau_{1}, \tau_{2}, \mathcal{J}\right)$ is said to be pairwise $\mathcal{I}$-normal if for each $\tau_{i}$-closed set $F$, each $\tau_{j}$-closed set $G$, with $i \neq j$ and $F \cap G=\varnothing$, there are disjoint $U \in \tau_{j}$ and $V \in \tau_{i}$ such that $F \backslash U \in \mathcal{J}$ and $G \backslash V \in \mathcal{J}$.

It is clear that if $\left(X, \tau_{1}, \tau_{2}\right)$ is pairwise normal then $\left(X, \tau_{1}, \tau_{2}, \mathcal{J}\right)$ is pairwise $\mathcal{I}$-normal, and that $\left(X, \tau_{1}, \tau_{2}\right)$ is pairwise normal if and only if $\left(X, \tau_{1}, \tau_{2},\{\varnothing\}\right)$ is pairwise $\mathcal{I}$-normal. It is also clear that if $\left(X, \tau_{1}, \tau_{2}, \mathcal{J}\right)$ is pairwise $\mathcal{I}$-normal and $A$ is a $\tau_{i}$-closed subset of $X$, for each $i \in\{1,2\}$, then $\left(A,\left(\tau_{1}\right)_{A},\left(\tau_{2}\right)_{A}, \mathcal{J}_{A}\right)$ is pairwise $\mathcal{I}$-normal.

Example 3.2 1) The space $(\mathbf{R}, \mathcal{C}, \gamma, \mathcal{J})$, where $\mathcal{J}$ is the ideal of all upper bounded subsets of $\mathbf{R}$, is pairwise $\mathcal{I}$-normal because if $F$ is a nonempty $\mathcal{C}$-closed set, $G$ is a nonempty $\gamma$-closed set and if $F \cap G=\varnothing$, then there is a $a \in \mathbf{R}$ such that $F=(-\infty, a]$. If we do $U=(-\infty, a)$ and $V=(a, \infty)$ then $G \backslash V \in \mathcal{J}, F \backslash U \in \mathcal{J}, U \in \gamma, V \in \mathcal{C}$ and $U \cap V=\varnothing$.

However $(\mathbf{R}, \mathcal{C}, \gamma)$ is not pairwise normal given that if we consider the $\mathcal{C}$-closed set $F=(-\infty, 0]$ and the $\gamma$-closed set $G=\left\{\frac{1}{n}: n \in Z^{+}\right\}$then, for 
each $U \in \gamma$ and $V \in \mathcal{C}$, if $F \subseteq U$ and $G \subseteq V$, then $U \cap V \neq \varnothing$.

2) If $\mathcal{P}_{f}(\mathbf{R} \backslash \mathbf{Q})$ is the set of all finite subsets of $\mathbf{R} \backslash \mathbf{Q}$, then the space $\left(\mathbf{R}, \mathcal{C}, \gamma, \mathcal{P}_{f}(\mathbf{R} \backslash \mathbf{Q})\right)$ is not pairwise $\mathcal{I}$-normal. Consider the $\mathcal{C}$-closed set $F=(-\infty, 0]$ and the $\gamma$-closed set $G=\left\{1 / n: n \in \mathbf{Z}^{+}\right\}$. If $V \in \mathcal{C}$ and $G \backslash V \in \mathcal{P}_{f}(\mathbf{R} \backslash \mathbf{Q})$ then the only option is that $G \subseteq V$, and this implies that $(0, \infty) \subseteq V$. Now, if $U \in \gamma$ and $U \cap V=\varnothing$, we have that $U \subseteq(-\infty, 0]$. Since $U \in \gamma$ then $0 \in \mathbf{R} \backslash U$, and so $F \backslash U \notin \mathcal{P}_{f}(\mathbf{R} \backslash \mathbf{Q})$.

In the following theorem we present some characterizations of pairwise $\mathcal{I}$-normality.

Theorem 3.3 The following propositions are equivalent: 1) The space $\left(X, \tau_{1}, \tau_{2}, \mathcal{J}\right)$ is pairwise $\mathcal{I}$-normal. 2) For each $i \neq j$, if $F$ is $\tau_{i}$ -closed, $W \in \tau_{j}$ and $F \subseteq W$, then there are $U \in \tau_{j}$ and a $\tau_{i}$-closed set $G$, such that $F \backslash U \in \mathcal{J}, \quad U \subseteq G$ and $G \backslash W \in \mathcal{J}$. 3) For each $i$ $\neq j$, if $F$ is $\tau_{i}$-closed, $W \in \tau_{j}$ and $F \subseteq W$, then there is a $U \in \tau_{j}$ such that $\left\{F \backslash U, a d h_{\tau_{i}}(U) \backslash W\right\} \subseteq \mathcal{J}$. 4) For each $i \neq j$, if $F$ is $\tau_{i}$ -closed, $G$ is $\tau_{j}$-closed and $F \cap G=\varnothing$, then there is a $U \in \tau_{j}$ such that $\left\{F \backslash U, a d h_{\tau_{i}}(U) \cap G\right\} \subseteq \mathcal{J}$.

Proof. 1) $\rightarrow$ 2) Suppose that $i \neq j, F$ is $\tau_{i}$-closed, $W \in \tau_{j}$ and $F \subseteq W$. There are $U \in \tau_{j}$ and $V \in \tau_{i}$ such that $F \backslash U \in \mathcal{J},(X \backslash W) \backslash V \in \mathcal{J}$ and $U \cap V=\varnothing$. Hence $U \subseteq X \backslash V$ and $(X \backslash V) \backslash W \in \mathcal{J}$.

$2) \rightarrow 3$ ) Suppose that $i \neq j, F$ is $\tau_{i}$-closed, $W \in \tau_{j}$ and $F \subseteq W$. There are $U \in \tau_{j}$ and a $\tau_{i}$-closed set $G$ such that $F \backslash U \in \mathcal{J}, U \subseteq G$ and $G \backslash W \in \mathcal{J}$. Thus $a d h_{\tau_{i}}(U) \subseteq G$ and so $a d h_{\tau_{i}}(U) \backslash W \in \mathcal{J}$.

$3) \rightarrow 4)$ If $i \neq j, F$ is a $\tau_{i}$-closed set, $G$ is a $\tau_{j}$-closed set and $F \cap G=$ $\varnothing$, then there is a $U \in \tau_{j}$ such that $F \backslash U \in \mathcal{J}$ and $a d h_{\tau_{i}}(U) \backslash(X \backslash G) \in \mathcal{J}$. Then $a d h_{\tau_{i}}(U) \cap G \in \mathcal{J}$.

$4) \rightarrow 1$ ) If $i \neq j, F$ is a $\tau_{i}$-closed set, $G$ is a $\tau_{j}$-closed set and $F \cap G=\varnothing$, there exists $U \in \tau_{j}$ such that $\left\{F \backslash U, a d h_{\tau_{i}}(U) \cap G\right\} \subseteq \mathcal{J}$. In consequence $G \backslash\left(X \backslash a d h_{\tau_{i}}(U)\right) \in \mathcal{J}$.

Some functional properties of pairwise $\mathcal{I}$-normal spaces will be shown in the following two theorems.

Theorem 3.4 Suppose that, for each $i \in\{1,2\}, f:\left(X, \tau_{i}\right) \rightarrow\left(Y, \beta_{i}\right)$ is a continuous, closed and surjective function, that $\mathcal{J}$ is an ideal in $Y$ and that $\left(X, \tau_{1}, \tau_{2}, \mathcal{I}_{f, \mathcal{J}}\right)$ is pairwise $\mathcal{I}$-normal. Then $\left(Y, \beta_{1}, \beta_{2}, \mathcal{J}\right)$ is a pairwise $\mathcal{I}$-normal space. 
Proof. If $H$ is a $\beta_{i}$-closed set, $K$ is a $\beta_{j}$-closed set, with $i \neq j$ and $H \cap K=$ $\varnothing$, there are disjoint $U \in \tau_{j}$ and $V \in \tau_{i}$ such that $\left\{f^{-1}(H) \backslash U, f^{-1}(K) \backslash V\right\}$ $\subseteq \mathcal{I}_{f, \mathcal{J}}$. There is a $I \in \mathcal{J}$ with $f^{-1}(H) \backslash U \subseteq f^{-1}(I)$. Thus $f^{-1}(H \backslash I) \subseteq U$, $X \backslash U \subseteq f^{-1}[(Y \backslash H) \cup I], f(X \backslash U) \subseteq(Y \backslash H) \cup I, H \backslash I \subseteq Y \backslash f(X \backslash U)$, and so $H \backslash[Y \backslash f(X \backslash U)] \subseteq I$. This implies that $H \backslash[Y \backslash f(X \backslash U)] \in \mathcal{J}$. Similarly we obtain that $K \backslash[Y \backslash f(X \backslash V)] \in \mathcal{J}$. Moreover $Y \backslash f(X \backslash U) \in \beta_{j}, Y \backslash f(X \backslash V)$ $\in \beta_{i}$ and $[Y \backslash f(X \backslash U)] \cap[Y \backslash f(X \backslash V)]=\varnothing$, because $f$ is surjective and $U \cap V=\varnothing$.

Theorem 3.5 If $\left(Y, \beta_{1}, \beta_{2}, \mathcal{L}\right)$ is pairwise $\mathcal{I}$-normal and if, for each $i \in$ $\{1,2\}, f:\left(X, \tau_{i}\right) \rightarrow\left(Y, \beta_{i}\right)$ is a continuous, closed and injective function, then $\left(X, \tau_{1}, \tau_{2}, \mathcal{J}\right)$ is a pairwise $\mathcal{I}$-normal space, for each ideal $\mathcal{J}$ in $X$ with $f^{-1}(\mathcal{L}) \subseteq \mathcal{J}$.

Proof. If $F$ is a $\tau_{i}$-closed set, $G$ is a $\tau_{j}$-closed set, with $i \neq j$ and $F \cap G=\varnothing$, then there are disjoint $U \in \beta_{j}$ and $V \in \beta_{i}$ such that $\{f(F) \backslash U, f(G) \backslash V\} \subseteq$ $\mathcal{L}$. Hence $\left\{F \backslash f^{-1}(U), G \backslash f^{-1}(V)\right\} \subseteq f^{-1}(\mathcal{L}) \subseteq \mathcal{J}, f^{-1}(U) \in \tau_{j}, f^{-1}(V)$ $\in \tau_{i}$ and $f^{-1}(U) \cap f^{-1}(V)=\varnothing$.

Now we show a condition under which a pairwise $\mathcal{I}$-regular space is a pairwise $\mathcal{I}$-normal space.

Definition 3.6 The subset $F$ of the space $(X, \tau, \beta, \mathcal{J})$ is said to be $\mathcal{P}$ compact if for each cover $\left\{V_{\alpha}: \alpha \in \Delta\right\} \subseteq \tau \cup \beta$ of $F$, there exists a finite $\Delta_{0} \subseteq \Delta$ such that $F \backslash \bigcup_{\alpha \in \Delta_{0}} V_{\alpha} \in \mathcal{J}$. The space $(X, \tau, \beta, \mathcal{J})$ is defined to said $\mathcal{P}$-compact if $X$ is $\mathcal{P}$-compact.

It is clear that if $(X, \tau, \beta, \mathcal{J})$ is $\mathcal{P}$-compact, then $(X, \tau, \mathcal{J})$ and $(X, \beta, \mathcal{J})$ are $\mathcal{I}$-compact. Moreover, it is easy to see that if $(X, \tau, \beta, \mathcal{J})$ is $\mathcal{P}$-compact and $X \backslash F \in \tau \cup \beta$, then $F$ is $\mathcal{P}$-compact.

Example 3.7 1) If $\mathcal{J}=\{A \subseteq \mathbf{R}: A$ is upper bounded $\}$ then it is easy to see that the space $\left(\mathbf{R}, \mathcal{C}, C_{f}, \mathcal{J}\right)$ is $\mathcal{P}$-compact, where $C_{f}$ is the cofinite topology.

2) The sets $\tau_{1}=\{A \subseteq \mathbf{Z}$ : the proposition $0 \in A \Rightarrow 2 \mathbf{Z} \subseteq A$ is true and $\tau_{2}=\{A \subseteq \mathbf{Z}$ : the proposition $1 \in A \Rightarrow 2 \mathbf{Z} \subseteq A$ is true $\}$ are topologies in $\mathbf{Z}$, where $2 \mathbf{Z}$ is the set of even integers. Note that if $A \subseteq \mathbf{Z}$ and $0 \notin A$ then $A \in \tau_{1}$, and that if $B \subseteq \mathbf{Z}$ and $1 \notin B$ then $B \in \tau_{2}$. If $\mathcal{J}=\mathcal{P}(\mathbf{Z} \backslash 2 \mathbf{Z})$, then $\left(\mathbf{Z}, \tau_{1}, \mathcal{J}\right)$ and $\left(\mathbf{Z}, \tau_{2}, \mathcal{J}\right)$ are $\mathcal{I}$-compact. On the other hand, it is clear that, for each $n \in \mathbf{Z}, A_{n}=\{2 n+1\} \in \tau_{1}$, and $B_{n}=\{2 n\} \in \tau_{2}$. Then 
$\mathbf{Z}=\left(\bigcup_{n \in \mathbf{Z}} A_{n}\right) \cup\left(\bigcup_{n \in \mathbf{Z}} B_{n}\right)$, but there are no finite $\mathbf{Z}_{0} \subseteq \mathbf{Z}$ and $\mathbf{Z}_{1} \subseteq \mathbf{Z}$ such that $\mathbf{Z} \backslash\left[\left(\bigcup_{n \in \mathbf{Z}_{0}} A_{n}\right) \cup\left(\bigcup_{n \in \mathbf{Z}_{1}} B_{n}\right)\right] \in \mathcal{J}$. Hence $\left(\mathbf{Z}, \tau_{1}, \tau_{2}, \mathcal{J}\right)$ is not $\mathcal{P}$-compact.

Theorem 3.8 If $\left(X, \tau_{1}, \tau_{2}, \mathcal{J}\right)$ is a pairwise $\mathcal{I}$-regular and $\mathcal{P}$-compact space, then $\left(X, \tau_{1}, \tau_{2}, \mathcal{J}\right)$ is pairwise $\mathcal{I}$-normal.

Proof. Suppose that $F$ is a $\tau_{i}$-closed set, $G$ is a $\tau_{j}$-closed set, with $i \neq j$ and $F \cap G=\varnothing$. For each $f \in F$ there are disjoint $U_{f} \in \tau_{j}$ and $V_{f} \in \tau_{i}$, such that $f \in U_{f}$, and $G \backslash V_{f} \in \mathcal{J}$. Given that $F \subseteq \bigcup_{f \in F} U_{f}$ and $F$ is $\mathcal{P}$-compact, there is a finite $F_{0} \subseteq F$ with $F \backslash \bigcup_{f \in F_{0}} U_{f} \in \mathcal{J}$. If we do $U=\bigcup_{f \in F_{0}} U_{f}$ and $V$ $=\bigcap_{f \in F_{0}} V_{f}$ then $F \backslash U \in \mathcal{J}$ and $G \backslash V=\bigcup_{f \in F_{0}}\left(G \backslash V_{f}\right) \in \mathcal{J}$. Moreover $U \in \tau_{j}$, $V \in \tau_{i}$ and $U \cap V=\varnothing$.

We end this section to showing a property of pairwise $\mathcal{I}$-normality, related to $\tau \oplus \mathcal{J}$ topologies. Suppose that $(X, \tau, \mathcal{J})$ is an ideal space. Let $\mathcal{J}^{\otimes}=\mathcal{P}\left(\bigcup_{J \in \mathcal{J}} J\right)$ be. Note that $\mathcal{J} \subseteq \mathcal{J}^{\otimes}$ and that $\tau \oplus \mathcal{J}=\tau \oplus \mathcal{J}^{\otimes}=$ $\left\{V \cup I: V \in \tau\right.$ and $\left.I \in \mathcal{J}^{\otimes}\right\}$.

Theorem 3.9 If the space $(X, \tau \oplus \mathcal{J}, \beta \oplus \mathcal{J}, \mathcal{J})$ is pairwise $\mathcal{I}$-normal, then the space $\left(X, \tau, \beta, \mathcal{J}^{\otimes}\right)$ is pairwise $\mathcal{I}$-normal. So, if $(X, \tau \oplus \mathcal{J}, \beta \oplus \mathcal{J})$ is pairwise normal, then $\left(X, \tau, \beta, \mathcal{J}^{\otimes}\right)$ is pairwise $\mathcal{I}$-normal.

Proof. Suppose that $F$ is $\tau$-closed, $G$ is $\beta$-closed and $F \cap G=\varnothing$. Since $F$ is $(\tau \oplus \mathcal{J})$-closed and $G$ is $(\beta \oplus \mathcal{J})$-closed, there are disjoint $W \in \beta \oplus \mathcal{J}$ and $T \in \tau \oplus \mathcal{J}$ such that $F \backslash W \in \mathcal{J}$ and $G \backslash T \in \mathcal{J}$. Now, there exist $\left\{I_{1}, I_{2}\right\} \subseteq \mathcal{J}^{\otimes}, W_{1} \in \beta$ and $T_{1} \in \tau$ such that $W=W_{1} \cup I_{1}$ and $T=$ $T_{1} \cup I_{2}$. Thus $\left(F \backslash W_{1}\right) \backslash I_{1} \in \mathcal{J}$ and $\left(G \backslash T_{1}\right) \backslash I_{2} \in \mathcal{J}$, and in this way $\left\{F \backslash W_{1}, G \backslash T_{1}\right\} \subseteq \mathcal{J}^{\otimes}$, with $W_{1} \cap T_{1}=\varnothing$.

\section{Strongly pairwise $\mathcal{I}$-regular spaces}

We recall that a bitopological space $\left(X, \tau_{1}, \tau_{2}\right)$ is strongly pairwise regular [4] if for each $i \in\{1,2\}$, if $F$ is $\tau_{i}$-closed and $x \in X \backslash F$, then there are disjoint $V \in \tau_{i}$ and $U \in \tau_{j}$, with $j \neq i$, such that $F \subseteq i n t_{\tau_{i}}(U)$ and $x \in V$. 
Our purpose for this section is to present a generalization of this concept through ideals.

Definition 4.1 The ideal bitopological space $\left(X, \tau_{1}, \tau_{2}, \mathcal{J}\right)$ is said to be strongly pairwise $\mathcal{I}$-regular if for each $i \in\{1,2\}$, if $F$ is $\tau_{i}$-closed and $x$ $\in X \backslash F$, then there are disjoint $V \in \tau_{i}$ and $U \in \tau_{j}$, with $j \neq i$, such that $F \backslash i n t_{\tau_{i}}(U) \in \mathcal{J}$ and $x \in V$.

Example 4.21$)$ The space $(\mathbf{R}, \mathcal{C}, \mathcal{L}, \mathcal{J})$, where $\mathcal{J}$ is the ideal of all upper bounded subsets of $\mathbf{R}$, is strongly pairwise $\mathcal{I}$-regular, because:

a) If $F$ is $\mathcal{C}$-closed and $a \in \mathbf{R} \backslash F$, there is a $\alpha<a$ such that $(\alpha, \infty) \subseteq \mathbf{R} \backslash F$. If we do $U=\left(\frac{\alpha+a}{2}, \infty\right)$ and $V=\left(-\infty, \frac{\alpha+a}{2}\right)$, then $U \in \mathcal{C}, V \in \mathcal{L}, a \in U$, $F \backslash \operatorname{int}_{\mathcal{C}}(V)=F \in \mathcal{J}$ and $U \cap V=\varnothing$.

b) If $F$ is $\mathcal{L}$-closed and $a \in \mathbf{R} \backslash F$, there exists $b>a$ such that $F \subseteq \mathbf{R} \backslash[a, b)$. If we do $U=(b, \infty)$ and $V=[a, b)$, then $U \in \mathcal{C}, V \in \mathcal{L}, a \in V, F \backslash$ int $_{\mathcal{L}}(U)$ $=F \backslash U \in \mathcal{J}$ and $U \cap V=\varnothing$.

However, the space $(\mathbf{R}, \mathcal{C}, \mathcal{L})$ is not strongly pairwise regular since there are no disjoint $U \in \mathcal{L}$ and $V \in \mathcal{C}$ such that $(-\infty, 0] \subseteq i n t_{\mathcal{C}}(U)$ and $1 \in V$. 2) Suppose that $X=\{0,1,2,3,4\}, \tau=\{\varnothing, X,\{0,1,2\},\{2,3,4\},\{2\}\}$, $\beta=\{\varnothing, X,\{3\},\{0,1\},\{0,1,3\}\}$ and $\mathcal{J}=\{\varnothing,\{3\},\{4\},\{3,4\}\}$, then it is easy to check that $(X, \tau, \beta, \mathcal{J})$ is a pairwise $\mathcal{I}$-regular space. On the other hand, this space is not strongly pairwise $\mathcal{I}$-regular given that $G=\{0,1,2,4\}$ is a $\beta$-closed set and $3 \in X \backslash G$, but if $U \in \tau, V \in \beta, 3 \in V$ and $U \cap V=\varnothing$, then the only options are as follows:

a) $U=\varnothing$ and $V=\{3\}$, or $U=\varnothing$ and $V=\{0,1,3\}$. In this cases $G \backslash \operatorname{int}_{\beta}(U)=G \notin \mathcal{J}$.

b) $U=\{0,1,2\}$ and $V=\{3\}$. In this case $G \backslash \operatorname{int}_{\beta}(U)=\{2,4\} \notin \mathcal{J}$.

c) $U=\{2\}$ and $V=\{3\}$, or $U=\{2\}$ and $V=\{0,1,3\}$. In this cases $G \backslash \operatorname{int}_{\beta}(U)=G \notin \mathcal{J}$.

Thus we have the following diagram, where all the implications are not revertible. 


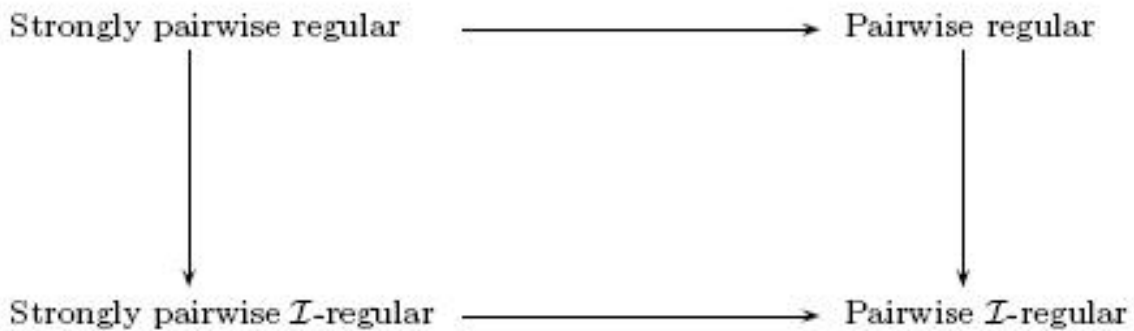

A couple of characterizations of strongly pairwise $\mathcal{I}$-regular spaces will be shown in the next two theorems.

Theorem 4.3 The space $\left(X, \tau_{1}, \tau_{2}, \mathcal{J}\right)$ is strongly pairwise $\mathcal{I}$-regular if, and only if, for each $i \in\{1,2\}, U \in \tau_{i}$, and $x \in U$, there are $W \in \tau_{i}$ and $X \backslash C \in \tau_{j}$, with $j \neq i$, such that $x \in W \subseteq C$ and $a d h_{\tau_{i}}(C) \backslash U \in \mathcal{J}$.

Proof. $(\rightarrow)$ Suppose that $i \in\{1,2\}, U \in \tau_{i}$ and $x \in U$. Since $\left(X, \tau_{1}, \tau_{2}, \mathcal{J}\right)$ is strongly pairwise $\mathcal{I}$-regular, there are disjoint sets $W \in \tau_{i}$ and $V \in \tau_{j}$, with $i \neq j$, such that $x \in W$ and $(X \backslash U) \backslash i n t_{\tau_{i}}(V) \in \mathcal{J}$. Thus $W \subseteq X \backslash V$ and $a d h_{\tau_{i}}(X \backslash V) \backslash U=(X \backslash U) \backslash i n t_{\tau_{i}}(V) \in \mathcal{J}$.

$(\leftarrow)$ Suppose that $i \in\{1,2\}, X \backslash F \in \tau_{i}$ and $x \in X \backslash F$. Our hypothesis implies that, if $j \neq i$, there are $W \in \tau_{i}$ and $X \backslash C \in \tau_{j}$ such that $x \in W \subseteq C$ and $a d h_{\tau_{i}}(C) \backslash(X \backslash F) \in \mathcal{J}$. So, $F \backslash i n t_{\tau_{i}}(X \backslash C) \in \mathcal{J}$ and $W \cap(X \backslash C)=\varnothing$.

Theorem 4.4 The space $\left(X, \tau_{1}, \tau_{2}, \mathcal{J}\right)$ is strongly pairwise $\mathcal{I}$-regular if, and only if, for each $i \in\{1,2\}, U \in \tau_{i}$, and $x \in U$, there is a $W \in \tau_{i}$ such that $x \in W$ and $a d h_{\tau_{i}}\left(a d h_{\tau_{j}}(W)\right) \backslash U \in \mathcal{J}$, where $j \neq i$.

Proof. $(\rightarrow)$ Suppose that $\left(X, \tau_{1}, \tau_{2}, \mathcal{J}\right)$ is strongly pairwise $\mathcal{I}$-regular, $i$ $\in\{1,2\}, U \in \tau_{i}$ and $x \in U$. By Theorem 4.3, there are $W \in \tau_{i}$ and $X \backslash C \in \tau_{j}$, with $j \neq i$, such that $x \in W \subseteq C$ and $a d h_{\tau_{i}}(C) \backslash U \in \mathcal{J}$. Since $a d h_{\tau_{j}}(W) \subseteq C$ then $a d h_{\tau_{i}}\left(a d h_{\tau_{j}}(W)\right) \subseteq a d h_{\tau_{i}}(C)$. Consequently $a d h_{\tau_{i}}\left(a d h_{\tau_{j}}(W)\right) \backslash U \in \mathcal{J}$.

$(\leftarrow)$ If $i \in\{1,2\}, X \backslash F \in \tau_{i}$ and $x \in X \backslash F$, then there is a $W \in \tau_{i}$ such that $x \in W$ and $a d h_{\tau_{i}}\left(a d h_{\tau_{j}}(W)\right) \backslash(X \backslash F) \in \mathcal{J}$. Hence $F \backslash i n t_{\tau_{i}}\left(X \backslash a d h_{\tau_{j}}(W)\right)$ 
$\in \mathcal{J}$. Moreover $W \cap\left(X \backslash a d h_{\tau_{j}}(W)\right)=\varnothing$. In this way $\left(X, \tau_{1}, \tau_{2}, \mathcal{J}\right)$ is a strongly pairwise $\mathcal{I}$-regular space.

Here a relationship between strongly pairwise $\mathcal{I}$-regularity and compactness.

Theorem 4.5 If the space $\left(X, \tau_{1}, \tau_{2}, \mathcal{J}\right)$ is strongly pairwise $\mathcal{I}$-regular then, for all $i \in\{1,2\}$, if $X \backslash F \in \tau_{i}$ and $K$ is compact in $\left(X, \tau_{i}\right)$, with $F \cap K=\varnothing$, then there are disjoint $W \in \tau_{i}$ and $V \in \tau_{j}$, with $j \neq i$, such that $K \subseteq W$ and $F \backslash i n t_{\tau_{i}}(V) \in \mathcal{J}$.

Proof. Suppose that $i \in\{1,2\}, X \backslash F \in \tau_{i}, K$ is compact in $\left(X, \tau_{i}\right)$ and $F \cap K=\varnothing$. For each $x \in K$, there are disjoint $W_{x} \in \tau_{i}$ and $V_{x} \in \tau_{j}$, with $j \neq i$, such that $x \in W_{x}$ and $F \backslash i n t_{\tau_{i}}\left(V_{x}\right) \in \mathcal{J}$. Given that $K$ is compact in $\left(X, \tau_{i}\right)$, there is a finite $K_{0} \subseteq K$ such that $K \subseteq W=\bigcup_{x \in K_{0}} W_{x}$. If we do $V=\bigcap_{x \in K_{0}} V_{x}$ then $V \cap W=\varnothing$ and $F \backslash \operatorname{int}_{\tau_{i}}(V)=\bigcup_{x \in K_{0}}\left(F \backslash i n t_{\tau_{i}}\left(V_{x}\right)\right) \in \mathcal{J}$.

Next three theorems show us some functional properties of strongly pairwise $\mathcal{I}$-regular spaces.

Theorem 4.6 If for each $i \in\{1,2\}$ the function $f:\left(X, \tau_{i}\right) \rightarrow\left(Y, \beta_{i}\right)$ is continuous, open, closed and surjective, and if $\left(X, \tau_{1}, \tau_{2}, \mathcal{J}\right)$ is strongly pairwise $\mathcal{I}$-regular, then the space $\left(Y, \beta_{1}, \beta_{2}, f(\mathcal{J})\right)$ is strongly pairwise $\mathcal{I}$-regular.

Proof. If $i \in\{1,2\}, Y \backslash G \in \beta_{i}$ and $b=f(a) \in Y \backslash G$ then there are $J$ $\in \mathcal{J}$ and disjoint $W \in \tau_{i}$ and $V \in \tau_{j}$, with $j \neq i$, such that $a \in W$ and $f^{-1}(G) \backslash i n t_{\tau_{i}}(V)=J$. If we do $T=\operatorname{int}_{\tau_{i}}(V) \cup J$, then $f^{-1}(G) \subseteq T$ and so $G \subseteq Y \backslash f(X \backslash T)=Y \backslash f\left[a d h_{\tau_{i}}(X \backslash V) \backslash J\right] \subseteq Y \backslash\left[f\left(a d h_{\tau_{i}}(X \backslash V)\right) \backslash f(J)\right]$ $=\left[Y \backslash f\left(a d h_{\tau_{i}}(X \backslash V)\right)\right] \cup f(J)=\left[Y \backslash a d h_{\beta_{i}}(f(X \backslash V))\right] \cup f(J)$, because $f:$ $\left(X, \tau_{i}\right) \rightarrow\left(Y, \beta_{i}\right)$ is closed and continuous. In this way $G \backslash \operatorname{int}_{\beta_{i}}[Y \backslash f(X \backslash V)]$ $\subseteq f(J)$. Thus $G \backslash \operatorname{int}_{\beta_{i}}[Y \backslash f(X \backslash V)] \in f(\mathcal{J})$. Furthermore, since $W \subseteq$ $X \backslash V$, we have that $Y \backslash f[X \backslash V] \subseteq Y \backslash f(W)$, and so $f(W) \cap[Y \backslash f[X \backslash V]]=$ $\varnothing$.

Theorem 4.7 If $f:\left(X, \tau_{1}, \tau_{2}\right) \rightarrow\left(Y, \beta_{1}, \beta_{2}\right)$ is pairwise perfect, and if $\mathcal{L}$ is an ideal in $Y$ such that $\left(X, \tau_{1}, \tau_{2}, \mathcal{I}_{f, \mathcal{L}}\right)$ is a strongly pairwise $\mathcal{I}$-regular space, then $\left(Y, \beta_{1}, \beta_{2}, \mathcal{L}\right)$ is strongly pairwise $\mathcal{I}$-regular. 
Proof. Suppose that $i \in\{1,2\}, Y \backslash G \in \beta_{i}$ and $b \in Y \backslash G$. Since, in $\left(X, \tau_{i}\right)$, $f^{-1}(\{b\})$ is compact and $f^{-1}(G)$ is closed, and $f^{-1}(\{b\}) \cap f^{-1}(G)=\varnothing$, there are disjoint $W \in \tau_{i}$ and $V \in \tau_{j}$, with $j \neq i$, such that $f^{-1}(\{b\}) \subseteq W$ and $f^{-1}(G) \backslash i n t_{\tau_{i}}(V) \in \mathcal{I}_{f, \mathcal{L}}$, by Theorem 4.5. Hence $b \in Y \backslash f(X \backslash W)$ and there is a $L \in \mathcal{L}$ such that $f^{-1}(G) \backslash i n t_{\tau_{i}}(V) \subseteq f^{-1}(L)$. Proceeding as in the proof of Theorem 2.10, we obtain that $G \backslash\left[Y \backslash f\left(X \backslash i n t_{\tau_{i}}(V)\right)\right] \subseteq L$. But $Y \backslash f\left(X \backslash \operatorname{int}_{\tau_{i}}(V)\right)=Y \backslash f\left(a d h_{\tau_{i}}(X \backslash V)\right)=Y \backslash a d h_{\beta_{i}}(f(X \backslash V))$, because $f$ is continuous and closed. Thus $Y \backslash f\left(X \backslash \operatorname{int}_{\tau_{i}}(V)\right)=i n t_{\beta_{i}}(Y \backslash f(X \backslash V))$ and so $G \backslash \operatorname{int}_{\beta_{i}}(Y \backslash f(X \backslash V)) \in \mathcal{L}$. Finally, given that $f$ is surjective and $W \cap V$ $=\varnothing$, we have that $[Y \backslash f(X \backslash W)] \cap[Y \backslash f(X \backslash V)]=\varnothing$.

Theorem 4.8 If for each $i \in\{1,2\}$ we have that $f:\left(X, \tau_{i}\right) \rightarrow\left(Y, \beta_{i}\right)$ is a continuous, closed and injective function, and if $\left(Y, \beta_{1}, \beta_{2}, \mathcal{L}\right)$ is strongly pairwise $\mathcal{I}$-regular, then the space $\left(X, \tau_{1}, \tau_{2}, f^{-1}(\mathcal{L})\right)$ is strongly pairwise $\mathcal{I}$-regular.

Proof. Suppose that $i \in\{1,2\}, F$ is a $\tau_{i}$-closed set and that $a \in X \backslash F$. Given that $f(a) \notin f(F)$ and $f(F)$ is $\beta_{i}$-closed, there are disjoint $W \in \beta_{i}$ and $T \in \beta_{j}$, where $j \neq i$, such that $f(a) \in W$ and $f(F) \backslash \operatorname{int}_{\beta_{i}}(T) \in \mathcal{L}$. This implies that $a \in f^{-1}(W), F \backslash f^{-1}\left(\operatorname{int}_{\beta_{i}}(T)\right) \in f^{-1}(\mathcal{L}), f^{-1}(W) \in$ $\tau_{i}, f^{-1}(T) \in \tau_{j}$ and $f^{-1}(W) \cap f^{-1}(T)=\varnothing$. Moreover, given that $f$ is continuous we have that $F \backslash i n t_{\tau_{i}}\left(f^{-1}(T)\right) \subseteq F \backslash f^{-1}\left(\operatorname{int}_{\beta_{i}}(T)\right)$, and so $F \backslash \operatorname{int}_{\tau_{i}}\left(f^{-1}(T)\right) \in f^{-1}(\mathcal{L})$.

We end this section by examining the products of strongly pairwise $\mathcal{I}$-regular spaces.

Theorem 4.9 If $\left(X, \tau_{1}, \tau_{2}, \mathcal{J}\right)$ and $\left(Y, \beta_{1}, \beta_{2}, \mathcal{L}\right)$ are strongly pairwise $\mathcal{I}$ -regular spaces, then $\left(X \times Y, \mu_{1}, \mu_{2}, \mathcal{J} \otimes \mathcal{L}\right)$ is strongly pairwise $\mathcal{I}$-regular, where $\mu_{i}=\tau_{i} \times \beta_{i}$, for each $i \in\{1,2\}$.

Proof. Suppose that $i \in\{1,2\}, W \in \tau_{i} \times \beta_{i}$ and $(a, b) \in W$. There are $U \in$ $\tau_{i}$ and $V \in \beta_{i}$ such that $(a, b) \in U \times V \subseteq W$. Now, by Theorem 4.4, there are $U_{1} \in \tau_{i}$ and $V_{1} \in \beta_{i}$, such that $a \in U_{1}, b \in V_{1}, a d h_{\tau_{i}}\left(a d h_{\tau_{j}}\left(U_{1}\right)\right) \backslash U \in \mathcal{J}$ and $a d h_{\beta_{i}}\left(a d h_{\beta_{j}}\left(V_{1}\right)\right) \backslash V \in \mathcal{L}$, where $j \neq i$. Let $J=a d h_{\tau_{i}}\left(a d h_{\tau_{j}}\left(U_{1}\right)\right) \backslash U$ and $L=a d h_{\beta_{i}}\left(a d h_{\beta_{j}}\left(V_{1}\right)\right) \backslash V$.

So $a d h_{\mu_{i}}\left(a d h_{\mu_{j}}\left(U_{1} \times V_{1}\right)\right) \backslash W \subseteq a d h_{\mu_{i}}\left(a d h_{\mu_{j}}\left(U_{1} \times V_{1}\right)\right) \backslash(U \times V)=$ $\left[J \times a d h_{\beta_{i}}\left(a d h_{\beta_{j}}\left(V_{1}\right)\right)\right] \cup\left[a d h_{\tau_{i}}\left(a d h_{\tau_{j}}\left(U_{1}\right)\right) \times L\right]$, and in this way we obtain that $a d h_{\mu_{i}}\left(a d h_{\mu_{j}}\left(U_{1} \times V_{1}\right)\right) \backslash W \in \mathcal{J} \otimes \mathcal{L}$. 
Hence Theorem 4.4 implies that $\left(X \times Y, \mu_{1}, \mu_{2}, \mathcal{J} \otimes \mathcal{L}\right)$ is strongly pairwise $\mathcal{I}$-regular.

Theorem 4.10 If $\left\{\left(X_{\alpha}, \tau_{1 \alpha}, \tau_{2 \alpha}, \mathcal{J}_{\alpha}\right): \alpha \in \Delta\right\}$ is a collection of strongly pairwise $\mathcal{I}$-regular spaces, then $\left(\prod_{\alpha \in \Delta} X_{\alpha}, \prod_{\alpha \in \Delta} \tau_{1 \alpha}, \prod_{\alpha \in \Delta} \tau_{2 \alpha}, \underset{\alpha \in \Delta}{\otimes} \mathcal{J}_{\alpha}\right)$ is strongly pairwise $\mathcal{I}$-regular.

Proof. To begin, we define $X=\prod_{\alpha \in \Delta} X_{\alpha}, \tau_{1}=\prod_{\alpha \in \Delta} \tau_{1 \alpha}$ and $\tau_{2}=\prod_{\alpha \in \Delta} \tau_{2 \alpha}$ and $\mathcal{J}=\bigotimes_{\alpha \in \Delta} \mathcal{J}_{\alpha}$

Suppose that $i \in\{1,2\}, W \in \tau_{i}$ and $a=\left(a_{\alpha}\right)_{\alpha \in \Delta} \in W$. There are $\left\{\alpha_{1}, \alpha_{2}, \ldots, \alpha_{r}\right\} \subseteq \Delta$ and $V_{\alpha_{j}} \in \tau_{i \alpha_{j}}$, for each $j \in\{1,2, \ldots, r\}$, such that $a$ $\in \bigcap_{j=1}^{r} p_{\alpha_{j}}^{-1}\left(V_{\alpha_{j}}\right) \subseteq W$, where $p_{\alpha_{j}}$ is the $\alpha_{j}$-th projection. If $j \in\{1,2, \ldots, r\}$ then $a_{\alpha_{j}} \in V_{\alpha_{j}}$, and so, by Theorem 4.4, there is a $U_{\alpha_{j}} \in \tau_{i \alpha_{j}}$ such that $a_{\alpha_{j}} \in U_{\alpha_{j}}$ and $a d h_{\tau_{i \alpha_{j}}}\left(a d h_{\tau_{k \alpha_{j}}}\left(U_{\alpha_{j}}\right)\right) \backslash V_{\alpha_{j}} \in \mathcal{J}_{\alpha_{j}}$, where $k \neq i$. Now, if we do $U=\bigcap_{j=1}^{r} p_{\alpha_{j}}^{-1}\left(U_{\alpha_{j}}\right)$ then $U \in \tau_{i}$ and $a \in U$. If $j \in\{1,2, \ldots, r\}$ we have that $a d h_{\tau_{k}}\left(p_{\alpha_{j}}^{-1}\left(U_{\alpha_{j}}\right)\right) \subseteq p_{\alpha_{j}}^{-1}\left(a d h_{\tau_{k \alpha_{j}}}\left(U_{\alpha_{j}}\right)\right) \subseteq p_{\alpha_{j}}^{-1}\left[a d h_{\tau_{i \alpha_{j}}}\left(a d h_{\tau_{k \alpha_{j}}}\left(U_{\alpha_{j}}\right)\right)\right]$, and then $a d h_{\tau_{i}}\left[a d h_{\tau_{k}}\left(p_{\alpha_{j}}^{-1}\left(U_{\alpha_{j}}\right)\right)\right] \subseteq p_{\alpha_{j}}^{-1}\left[a d h_{\tau_{i \alpha_{j}}}\left(a d h_{\tau_{k \alpha_{j}}}\left(U_{\alpha_{j}}\right)\right)\right]$.

So $a d h_{\tau_{i}}\left(a d h_{\tau_{k}}(U)\right) \backslash W \subseteq \bigcap_{j=1}^{r} a d h_{\tau_{i}}\left[a d h_{\tau_{k}}\left(p_{\alpha_{j}}^{-1}\left(U_{\alpha_{j}}\right)\right)\right] \backslash \bigcap_{j=1}^{r} p_{\alpha_{j}}^{-1}\left(V_{\alpha_{j}}\right)$ $\subseteq \bigcap_{j=1}^{r} p_{\alpha_{j}}^{-1}\left[a d h_{\tau_{i \alpha_{j}}}\left(a d h_{\tau_{k \alpha_{j}}}\left(U_{\alpha_{j}}\right)\right)\right] \backslash \bigcap_{j=1}^{r} p_{\alpha_{j}}^{-1}\left(V_{\alpha_{j}}\right)$.

Let $A=\bigcap_{j=1}^{r} p_{\alpha_{j}}^{-1}\left[a d h_{\tau_{i \alpha_{j}}}\left(a d h_{\tau_{k \alpha_{j}}}\left(U_{\alpha_{j}}\right)\right)\right] \backslash \bigcap_{j=1}^{r} p_{\alpha_{j}}^{-1}\left(V_{\alpha_{j}}\right)$ be. Given that, for each $j \in\{1,2, \ldots, r\}, p_{\alpha_{j}}^{-1}\left[a d h_{\tau_{i \alpha_{j}}}\left(a d h_{\tau_{k \alpha_{j}}}\left(U_{\alpha_{j}}\right)\right) \backslash V_{\alpha_{j}}\right] \in \mathcal{J}$ and $A \subseteq \bigcup_{j=1}^{r} p_{\alpha_{j}}^{-1}\left[a d h_{\tau_{i \alpha_{j}}}\left(a d h_{\tau_{k \alpha_{j}}}\left(U_{\alpha_{j}}\right)\right) \backslash V_{\alpha_{j}}\right]$, then $a d h_{\tau_{i}}\left[a d h_{\tau_{k}}(U)\right] \backslash W \in$ $\mathcal{J}$.

Hence $\left(X, \tau_{1}, \tau_{2}, \mathcal{J}\right)$ is strongly pairwise $\mathcal{I}$-regular.

\section{Strongly pairwise $\mathcal{I}$-normal spaces.}

A bitopological space $\left(X, \tau_{1}, \tau_{2}\right)$ is said to be strongly pairwise normal [4] if for each $\tau_{i}$-closed set $F$, each $\tau_{j}$-closed set $G$, with $i \neq j$ and $F \cap G$ $=\varnothing$, there are disjoint $U \in \tau_{j}$ and $V \in \tau_{i}$ such that $F \subseteq i n t_{\tau_{i}}(U)$ and 
$G \subseteq i n t_{\tau_{j}}(V)$. Our interest for this section is to introduce a generalization of this concept, through ideals.

Definition 5.1 The ideal bitopological space $\left(X, \tau_{1}, \tau_{2}, \mathcal{J}\right)$ is said to be strongly pairwise $\mathcal{I}$-normal if for each $\tau_{i}$-closed set $F$, each $\tau_{j}$-closed set $G$, with $i \neq j$ and $F \cap G=\varnothing$, there are disjoint $U \in \tau_{j}$ and $V \in \tau_{i}$ such that $\left\{F \backslash i n t_{\tau_{i}}(U), G \backslash i n t_{\tau_{j}}(V)\right\} \subseteq \mathcal{J}$.

Example 5.1 1) If $X=\{0,1,2\}, \tau=\{\varnothing, X,\{0\},\{1\},\{0,1\},\{0,2\}\}$, $\beta=\{\varnothing, X,\{0\},\{2\},\{0,2\},\{1,2\}\}$ and $\mathcal{J}=\mathcal{P}(\{1,2\})$, then $(X, \tau, \beta, \mathcal{J})$ is a strongly pairwise $\mathcal{I}$-normal space, because $\{1,2\} \backslash$ int $_{\tau}(\{1,2\})=\{2\}$ and $\{0\} \backslash \operatorname{int}_{\beta}(\{0\})=\varnothing,\{0,2\} \backslash \operatorname{int}_{\tau}(\{0,2\})=\varnothing$ and $\{1\} \backslash \operatorname{int}_{\beta}(\varnothing)=\{1\}$, $\{2\} \backslash \operatorname{int}_{\tau}(\varnothing)=\{2\}$ and $\{0,1\} \backslash \operatorname{int}_{\beta}(\{0\})=\{1\},\{2\} \backslash \operatorname{int}_{\tau}(\varnothing)=\{2\}$ and $\{1\} \backslash \operatorname{int}_{\beta}(\varnothing)=\{1\},\{2\} \backslash \operatorname{int}_{\tau}(\varnothing)=\{2\}$ and $\{0\} \backslash \operatorname{int}_{\beta}(\{0\})=\varnothing$, and finally, $\{1\} \backslash \operatorname{int}_{\tau}(\varnothing)=\{1\}$ and $\{0\} \backslash \operatorname{int}_{\beta}(\{0\})=\varnothing$.

However, the space $(X, \tau, \beta)$ is not strongly pairwise normal, because if $U \in \beta$ and $\{1,2\} \subseteq \operatorname{int}_{\tau}(U)$, then $U=X$ and so, if $V \in \tau$ and $\{0\} \subseteq \operatorname{int}_{\beta}(V)$, we have that $U \cap V=V \neq \varnothing$.

2) The space $\left(\mathbf{R}, \mathcal{C}, \mathcal{L}, \mathcal{I}_{f}(\mathbf{R})\right)$ is not strongly pairwise $\mathcal{I}$-normal, because the set $F=(-\infty, 0]$ is $\mathcal{C}$-closed, the set $G=[1, \infty)$ is $\mathcal{L}$-closed, and if $U \in \mathcal{L}, V \in \mathcal{C}$ are disjoint sets and $F \backslash \operatorname{int}_{\mathcal{C}}(U) \in \mathcal{I}_{f}(\mathbf{R})$, then we must have that $\operatorname{int}_{\mathcal{C}}(U)=\mathbf{R}$ and so $U=\mathbf{R}, V=\varnothing$ and $G \backslash i n t_{\mathcal{L}}(V)=G \notin \mathcal{I}_{f}(\mathbf{R})$.

On the other hand, the space $\left(\mathbf{R}, \mathcal{C}, \mathcal{L}, \mathcal{I}_{f}(\mathbf{R})\right)$ is pairwise $\mathcal{I}$-normal since, if we have a nonempty $\mathcal{C}$-closed set $F$, and a nonempty $\mathcal{L}$-closed set $G$, with $F \cap G=\varnothing$, then there is a $a \in \mathbf{R}$ such that $F=(-\infty, a]$. If we do $U=(-\infty, a)$ and $V=(a, \infty)$ then $F \backslash U=\{a\} \in \mathcal{I}_{f}(\mathbf{R})$ and $G \backslash V=\varnothing \in \mathcal{I}_{f}(\mathbf{R})$.

Hence we have the following diagram, where all the implications are not revertible. 


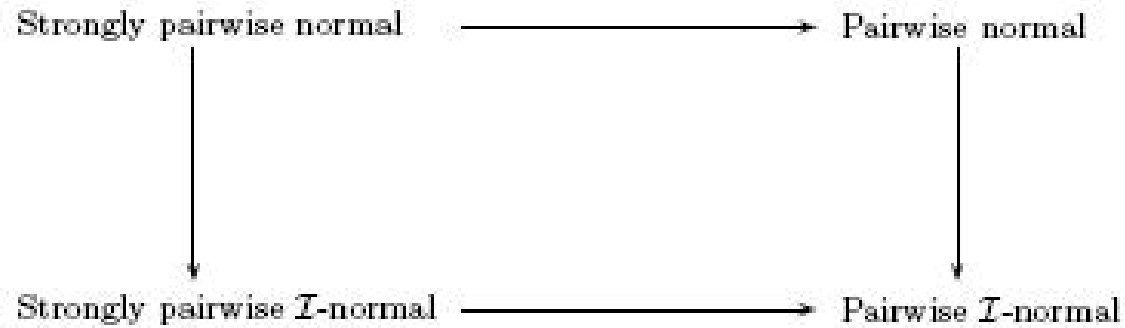

Theorem 5.2 If $\left(X, \tau_{1}, \tau_{2}, \mathcal{J}\right)$ is a strongly pairwise $\mathcal{I}$-normal space and if $A$ is $\tau_{i}$-closed, for each $i \in\{1,2\}$, then $\left(A,\left(\tau_{1}\right)_{A},\left(\tau_{2}\right)_{A}, \mathcal{J}_{A}\right)$ is strongly pairwise $\mathcal{I}$-normal.

Proof. Suppose that $F$ is $\left(\tau_{i}\right)_{A^{-c l o s e d},} G$ is $\left(\tau_{j}\right)_{A^{-c l o s e d}}$, with $i \neq j$ and $F \cap G=\varnothing$. Given that $F$ is $\tau_{i}$-closed and $G$ is $\tau_{j}$-closed, there are disjoint $U \in \tau_{j}$ and $V \in \tau_{i}$ such that $\left\{F \backslash i n t_{\tau_{i}}(U), G \backslash i n t_{\tau_{j}}(V)\right\} \subseteq \mathcal{J}$. Given that $F \backslash i n t_{\tau_{i}}(U) \subseteq A$ and $G \backslash i n t_{\tau_{j}}(V) \subseteq A$, then $\left\{F \backslash i n t_{\tau_{i}}(U), G \backslash i n t_{\tau_{j}}(V)\right\} \subseteq$ $\mathcal{J}_{A}$. But $F \backslash i n t_{\tau_{i}}(U)=\varnothing \cup\left[F \backslash i n t_{\tau_{i}}(U)\right]=(F \backslash A) \cup\left[F \backslash i n t_{\tau_{i}}(U)\right]=$ $F \backslash\left[A \cap \operatorname{int}_{\tau_{i}}(U)\right]$. Similarly $G \backslash i n t_{\tau_{j}}(V)=G \backslash\left[A \cap i n t_{\tau_{j}}(V)\right]$.

Hence $\left\{F \backslash\left[A \cap i n t_{\tau_{i}}(U)\right], G \backslash\left[A \cap i n t_{\tau_{j}}(V)\right]\right\} \subseteq \mathcal{J}_{A}$. Now, since $A \cap$ $\operatorname{int}_{\tau_{i}}(U) \subseteq \operatorname{int}_{\left(\tau_{i}\right)_{A}}(A \cap U)$ and $A \cap \operatorname{int}_{\tau_{j}}(V) \subseteq i n t_{\left(\tau_{j}\right)_{A}}(A \cap V)$, we conclude that $F \backslash i n t_{\left(\tau_{i}\right)_{A}}(A \cap U) \in \mathcal{J}_{A}$ and $G \backslash i n t_{\left(\tau_{j}\right)_{A}}(A \cap V) \in \mathcal{J}_{A}$.

Here we have some interesting characterizations of strongly pairwise $\mathcal{I}$-normality.

Theorem 5.3 For the ideal bitopological space $\left(X, \tau_{1}, \tau_{2}, \mathcal{J}\right)$, the following statements are equivalent:

1) $\left(X, \tau_{1}, \tau_{2}, \mathcal{J}\right)$ is strongly pairwise $\mathcal{I}$-normal.

2) For each $i \neq j$, if $F$ is $\tau_{i}$-closed, $U \in \tau_{j}$ and $F \subseteq U$, then there are $W \in \tau_{j}$ and a $\tau_{i}$-closed set $D$ such that $W \subseteq D$ and $\left\{F \backslash i n t_{\tau_{i}}(W), a d h_{\tau_{j}}(D) \backslash U\right\} \subseteq \mathcal{J}$. 
3) For each $i \neq j$, if $F$ is $\tau_{i}$-closed, $U \in \tau_{j}$ and $F \subseteq U$, then there exists $W \in \tau_{j}$ such that $\left\{F \backslash i n t_{\tau_{i}}(W), a d h_{\tau_{j}}\left(a d h_{\tau_{i}}(W)\right) \backslash U\right\} \subseteq \mathcal{J}$.

4) For each $i \neq j$, if $F$ is $\tau_{i}$-closed, $G$ is $\tau_{j}$-closed and $F \cap G=\varnothing$, there is a $W \in \tau_{j}$ such that $\left\{F \backslash i n t_{\tau_{i}}(W), G \cap a d h_{\tau_{j}}\left(a d h_{\tau_{i}}(W)\right)\right\} \subseteq \mathcal{J}$.

Proof.

$1) \rightarrow 2$ ) Suppose that $i \neq j, F$ is a $\tau_{i}$-closed set, $U \in \tau_{j}$ and $F \subseteq U$. There are disjoint $W \in \tau_{j}$ and $V \in \tau_{i}$ such that $F \backslash i n t_{\tau_{i}}(W) \in \mathcal{J}$ and $(X \backslash U) \backslash i n t_{\tau_{j}}(V) \in \mathcal{J}$. If we do $D=X \backslash V$ then $D$ is $\tau_{i}$-closed, $W \subseteq D$, $a d h_{\tau_{j}}(D) \backslash U=(X \backslash U) \backslash i n t_{\tau_{j}}(V) \in \mathcal{J}$.

$2) \rightarrow 3)$ Suppose that $i \neq j, F$ is $\tau_{i}$-closed, $U \in \tau_{j}$ and $F \subseteq U$. There are $W \in \tau_{j}$ and $X \backslash D \in \tau_{i}$ such that $W \subseteq D$ and $\left\{F \backslash i n t_{\tau_{i}}(W), a d h_{\tau_{j}}(D) \backslash U\right\}$ $\subseteq \mathcal{J}$. Hence $a d h_{\tau_{j}}\left(a d h_{\tau_{i}}(W)\right) \subseteq a d h_{\tau_{j}}(D)$, and so $a d h_{\tau_{j}}\left(a d h_{\tau_{i}}(W)\right) \backslash U \in$ $\mathcal{J}$.

3) $\rightarrow$ 4) This is clear.

$4) \rightarrow 1$ ) If $F$ is a $\tau_{1}$-closed set, $G$ is a $\tau_{2}$-closed and $F \cap G=\varnothing$, there is a $W \in \tau_{2}$ such that $\left\{F \backslash i n t_{\tau_{1}}(W), G \cap a d h_{\tau_{2}}\left(a d h_{\tau_{1}}(W)\right)\right\} \subseteq \mathcal{J}$. Then $G \backslash\left(X \backslash a d h_{\tau_{2}}\left(a d h_{\tau_{1}}(W)\right)\right) \in \mathcal{J}$, and so $G \backslash i n t_{\tau_{2}}\left(X \backslash a d h_{\tau_{1}}(W)\right) \in \mathcal{J}$. Moreover $W \cap\left(X \backslash a d h_{\tau_{1}}(W)\right)=\varnothing$.

Finally we present some functional properties of strongly pairwise $\mathcal{I}$ normality.

Theorem 5.4 Suppose that, for each $i \in\{1,2\}$, the function $f:\left(X, \tau_{i}\right) \rightarrow$ $\left(Y, \beta_{i}\right)$ is continuous, closed and surjective. If $\mathcal{J}$ is an ideal in $Y$ and the space $\left(X, \tau_{1}, \tau_{2}, \mathcal{I}_{f, \mathcal{J}}\right)$ is strongly pairwise $\mathcal{I}$-normal, then $\left(Y, \beta_{1}, \beta_{2}, \mathcal{J}\right)$ is strongly pairwise $\mathcal{I}$-normal.

Proof. Suppose that $H$ is a $\beta_{i}$-closed set, $K$ is $\beta_{j}$-closed, with $i \neq$ $j$ and $H \cap K=\varnothing$. There are disjoint $U \in \tau_{j}$ and $V \in \tau_{i}$ such that $f^{-1}(H) \backslash i n t_{\tau_{i}}(U) \in \mathcal{I}_{f, \mathcal{J}}$ and $f^{-1}(K) \backslash i n t_{\tau_{j}}(V) \in \mathcal{I}_{f, \mathcal{J}}$. There is a $J \in \mathcal{J}$ such that

$f^{-1}(H) \backslash i n t_{\tau_{i}}(U) \subseteq f^{-1}(J)$. Hence $f^{-1}(H \backslash J) \subseteq i n t_{\tau_{i}}(U), X \backslash i n t_{\tau_{i}}(U) \subseteq$ $f^{-1}((X \backslash H) \cup J), f\left(X \backslash \operatorname{int}_{\tau_{i}}(U)\right) \subseteq(X \backslash H) \cup J$, and so $H \subseteq\left[Y \backslash f\left(X \backslash \operatorname{int}_{\tau_{i}}(U)\right)\right] \cup$ $J$ or, equivalently, $H \subseteq\left[Y \backslash f\left(a d h_{\tau_{i}}(X \backslash U)\right)\right] \cup J$. But $a d h_{\beta_{i}}(f(X \backslash U))=$ $f\left(a d h_{\tau_{i}}(X \backslash U)\right)$, since $f:\left(X, \tau_{i}\right) \rightarrow\left(Y, \beta_{i}\right)$ is continuous and closed. 
Thus $H \subseteq\left[Y \backslash a d h_{\beta_{i}}(f((X \backslash U)))\right] \cup J$, and so $H \subseteq\left[\operatorname{int}_{\beta_{i}}(Y \backslash f((X \backslash U)))\right] \cup$ $J$. If we do $W=Y \backslash f(X \backslash U)$ then $H \backslash \operatorname{int}_{\beta_{i}}(W) \in \mathcal{J}$. Similarly, if we do $T=Y \backslash f(X \backslash V)$ then $K \backslash \operatorname{int}_{\beta_{j}}(T) \in \mathcal{J}$. Given that $f$ is surjective and $U \cap V=\varnothing$, we have that $W$ and $T$ are disjoint sets.

Consequently $\left(Y, \beta_{1}, \beta_{2}, \mathcal{J}\right)$ is a strongly pairwise $\mathcal{I}$-normal space.

Theorem 5.5 Suppose that, for each $i \in\{1,2\}$, the function $f:\left(X, \tau_{i}\right) \rightarrow$ $\left(Y, \beta_{i}\right)$ is one to one, continuous and closed. If the space $\left(Y, \beta_{1}, \beta_{2}, \mathcal{L}\right)$ is strongly pairwise $\mathcal{I}$-normal, then $\left(X, \tau_{1}, \tau_{2}, f^{-1}(\mathcal{L})\right)$ is strongly pairwise $\mathcal{I}$-normal.

Proof. Suppose that $F$ is $\tau_{i}$-closed, $G$ is $\tau_{j}$-closed, with $i=j$ and $F \cap G=$ $\varnothing$. There are disjoint $W \in \beta_{j}$ and $T \in \beta_{i}$ such that $f(F) \backslash \operatorname{int}_{\beta_{i}}(W) \in \mathcal{L}$ and $f(G) \backslash \operatorname{int}_{\beta_{j}}(T) \in \mathcal{L}$. This implies that $F \backslash f^{-1}\left(\operatorname{int}_{\beta_{i}}(W)\right) \in f^{-1}(\mathcal{L})$ and $G \backslash f^{-1}\left(\operatorname{int}_{\beta_{j}}(T)\right) \in f^{-1}(\mathcal{L})$.

But $f^{-1}\left(\operatorname{int}_{\beta_{i}}(W)\right) \subseteq \operatorname{int}_{\tau_{i}}\left(f^{-1}(W)\right)$ and $f^{-1}\left(\operatorname{int}_{\beta_{j}}(T)\right) \subseteq i n t_{\tau_{j}}\left(f^{-1}(T)\right)$, and so $\left\{F \backslash i n t_{\tau_{i}}\left(f^{-1}(W)\right), G \backslash i n t_{\tau_{j}}\left(f^{-1}(T)\right)\right\} \subseteq f^{-1}(\mathcal{L})$. On the other hand, $f^{-1}(W) \in \tau_{j}, f^{-1}(T) \in \tau_{i}$ and $f^{-1}(W) \cap f^{-1}(T)=\varnothing$.

\section{Acknowledgment:}

Research supported by Escuela Colombiana de Ingeniería Julio Garavito. The author wishes to express his gratitude to the anonymous referee, for his comments and suggestions, which are reflected in the final presentation of the paper.

\section{References}

[1] D. Jancovic and T.R. Hamlett, "New topologies from old via ideals", The American Mathematical Monthly, vol. 97, no. 4, pp. 295-310, Apr. 1990, doi: 10.1080/00029890.1990.11995593

[2] J. C. Kelly, "Bitopological spaces", Proceedings of the London Mathematical Society, vol. s3-13, no. 1, pp. 71-89, 1963, doi: 10.1112/ plms/ S3-13.1.71

[3] R. Newcomb, "Topologies which are compact modulo an ideal", Ph.D. dissertation, University of California at Santa Barbara, 1967. 
[4] A. Mukharjee, "Some new bitopological notions", Publications de l'Institut Mathematique, vol. 93, no 107, pp. 165-172, 2013, doi: 10.2298/ PIM1307165M

[5] N. R. Pachón Rubiano, "Between closed and Ig-closed sets", European journal of pure and applied mathematics, vol. 11, no. 1, pp. 299-314, Jan. 2018, doi: 10.29020/ nybg.ejpam.v11i2.3131

[6] N. R. Pachón Rubiano, "Some properties of J -Hausdorff, J -regular and $\mathcal{J}$ - normal spaces", Scientific studies and research, series Mathematics and informatics, vol. 28 no 2, pp. 49-62, 2018. [On line]. Available: https:/ / bit.ly/ 2ZZHDRe

[7] I. L. Reilly, "On bitopological separation properties", Nanta mathematica, vol. 29, pp. 14-25, 1972.

[8] R. Vaidyanathaswamy, "The localization theory in set-topology", Proceedings of the Indian Academy of Sciences, vol. 20, pp. 51-61, 1945, doi: 10.1007/ BF03048958 